University of Texas at El Paso

ScholarWorks@UTEP

$12-2008$

\title{
Intelligence Techniques Are Needed to Further Enhance the Advantage of Groups with Diversity in Problem Solving
}

Oscar Castillo

Patricia Melin

J. Esteban Gamez

Vladik Kreinovich

The University of Texas at El Paso, vladik@utep.edu

Olga Kosheleva

The University of Texas at El Paso, olgak@utep.edu

Follow this and additional works at: https://scholarworks.utep.edu/cs_techrep

Part of the Computer Engineering Commons

Comments:

Technical Report: UTEP-CS-08-37a

Published in the Proceedings of the 2009 IEEE Workshop on Hybrid Intelligent Models and Applications HIMA'2009, Nashville, Tennessee, March 30-April 2, 2009, pp. 48-55.

\section{Recommended Citation}

Castillo, Oscar; Melin, Patricia; Gamez, J. Esteban; Kreinovich, Vladik; and Kosheleva, Olga, "Intelligence Techniques Are Needed to Further Enhance the Advantage of Groups with Diversity in Problem Solving" (2008). Departmental Technical Reports (CS). 119.

https://scholarworks.utep.edu/cs_techrep/119

This Article is brought to you for free and open access by the Computer Science at ScholarWorks@UTEP. It has been accepted for inclusion in Departmental Technical Reports (CS) by an authorized administrator of ScholarWorks@UTEP.For more information, please contact Iweber@utep.edu. 


\title{
Intelligence Techniques Are Needed to Further Enhance the Advantage of Groups with Diversity in Problem Solving
}

\author{
Oscar Castillo, Patricia Melin, J. Esteban Gamez, Vladik Kreinovich, and Olga Kosheleva
}

\begin{abstract}
In practice, there are many examples when the diversity in a group enhances the group's ability to solve problems - and thus, leads to more efficient groups, firms, schools, etc. Several papers, starting with the pioneering research by Scott E. Page from the University of Michigan at Ann Arbor, provide a theoretical justification for this known empirical phenomenon. However, when the general advise of increasing diversity is transformed into simple-to-follow algorithmic rules (like quotas), the result is not always successful. In this paper, we prove that the problem of designing the most efficient group is computationally difficult (NP-hard). Thus, in general, it is not possible to come up with simple algorithmic rules for designing such groups: to design optimal groups, we need to combine standard optimization techniques with intelligent techniques that use expert knowledge.
\end{abstract}

\section{INTRODUCTION TO THE PROBLEM}

$\mathbf{I}^{\mathrm{N}}$ $\mathrm{N}$ real life, there are many examples that diversity in a group enhances the group's ability to solve problems - and thus, leads to more efficient groups, firms, schools, etc. Several papers, starting with the pioneering research by Scott E. Page from the University of Michigan at Ann Arbor, provide a theoretical justification for this known empirical phenomenon; see, e.g., [2], [3], [7], [9] and references therein. Specifically, these papers have shown that groups of diverse problem solvers can outperform groups of highability problem solvers.

The word can is here (and in the title of the paper [3]) for a good reason: when the general advise of increasing diversity is transformed into simple-to-follow algorithmic rules (like quotas), the result is not always successful.

In this paper, we consider the problem of designing the most efficient group as a precise optimization problem. We show that this optimization problem is computationally difficult (NP-hard). Thus, in general, it is not possible to come up with simple algorithmic rules for designing such groups: to design optimal groups, we need to combine standard optimization techniques with intelligent techniques that use expert knowledge.

Comment. Similar results are known: e.g., the problem of maximizing diversity and the problem of finding a group

Oscar Castillo and Patricia Melin are with Tijuana Institute of Technology, mailing address P.O. Box 4207, Chula Vista, CA 91909, USA (email \{ocastillo, epmelin\}@hafsamx.org). J. Esteban Gamez, Vladik Kreinovich (Department of Computer Science), and Olga Kosheleva (Department of Teacher Education) are with the University of Texas at El Paso, El Paso, TX 79968, USA (emails \{vladik, olgak\}@utep.edu).

This work was supported in part by NSF grant HRD-0734825 and by Grant 1 T36 GM078000-01 from the National Institutes of Health. The authors are thankful to the anonymous referees for valuable suggestions. which is most representative of the population are both NPhard [1], [6]. In this paper, we extent these results further - from gauging and maintaining the degree of diversity to gauging and maintaining the positive effects of diversity such as the increased ability of a group to solve problems.

\section{Towards the Formulation of the Problem IN EXACT TERMS}

Let us assume that we have a population consisting of $n$ individuals. From this population $\{1, \ldots, n\}$, we need to select a group $G \subseteq\{1, \ldots, n\}$ which is the most efficient in solving a given problem.

In mathematical terms, to describe a group $G$, we must describe, for each individual $i(i=1, \ldots, n)$, whether this individual is selected for this group or not. In computational terms, for each $i=1, \ldots, n$, we thus need to select a Boolean ("true"-"false") value $x_{i}$ for which:

- $x_{i}=$ "true" means that we select the $i$-th individual into the group, and

- $x_{i}=$ "false" means that we do not select the $i$-th individual into the group.

Inside the computer, "true" is usually represented as 1 , while "false" is usually represented as 0 . Thus, we can describe each group by selecting, for each $i=1, \ldots, n$, a value $x_{i} \in$ $\{0,1\}$ for which

- $x_{i}=1$ means that we select the $i$-th individual into the group, and

- $x_{i}=0$ means that we do not select the $i$-th individual into the group.

In order to select the most efficient group, we must describe how the group's efficiency $p$ depends on the selections $x_{i}$.

For simple mechanical tasks like digging trenches or doing simple menial work, people perform these tasks individually. For such tasks, the efficiency $p$ of a group is simply the sum of the productivity values $p_{i}$ of all the individuals who form this group $G$ :

$$
p=\sum_{i \in G} p_{i} .
$$

In terms of the variables $x_{i}$, this formula means that we add $p_{i}$ if $x_{i}=1$ and that we do not add $p_{i}$ if $x_{i}=0$. In other words, this formula can be described as

$$
p=\sum_{i=1}^{n} p_{i} \cdot x_{i} .
$$

In this simple model, the more people work on a project, the larger the productivity. 
Most practical problems are not that simple. In solving these problems, interaction between the individuals can enhance their productivity. In mathematical terms, this means that

- in addition to the above terms $p_{i} \cdot x_{i}$ which are linear in $x_{i}$,

- we also have terms

$$
p_{i j} \cdot x_{i} \cdot x_{j}, \quad i \neq j,
$$

which are quadratic in $x_{i}$; these terms describe pair-wise interaction between the individuals;

- we may also have cubic terms

$$
p_{i j k} \cdot x_{i} \cdot x_{j} \cdot x_{k}
$$

which describe triple interactions,

- and we can also have higher order terms, which describe the effect of larger subgroups.

In other words, in general, the formula describing the productivity of a group takes a more complex form

$$
p=\sum_{i=1}^{n} p_{i} \cdot x_{i}+\sum_{i \neq j} p_{i j} \cdot x_{i} \cdot x_{j}+\ldots
$$

For example, for a group consisting of two individuals, $i$ and $j$, the productivity is equal to

$$
p=p_{i}+p_{j}+p_{i j}+\ldots
$$

It should be mentioned that interaction is not always helpful. For example, if we are interested in solving a complex problem, and we bring together two individuals with similar ways of thinking and with similar skills, then there is not much that these individuals can learn from each other.

In some cases, they may speed up the process by dividing the testing of possible approaches between themselves. In such cases, they can solve the problem twice faster, the productivity increases twice - so there is, in effect, no interaction terms $p_{i j}$.

In other cases, when the problem is not easy to subdivide, the fact that we have two similar solvers solving the same problem does not help at all - the overall time is the same as for each individual solver. In this case, $p \approx p_{i} \approx p_{j}$ and thus $p<p_{i}+p_{j}$, i.e., $p_{i j}<0$.

On the hand, in a diverse group, individuals complement each other, learn from each other, and as a result, their productivity increases above what would have happened if they worked on their own: $p>p_{i}+p_{j}$, so $p_{i j}>0$.

Such "negative" and "positive" interactions (i.e., $p_{i j}<$ 0 and $p_{i j}>0$ ) are not just a negative possibility - this is exactly the reason why, as we have mentioned, groups of diverse problem solvers can outperform groups of highability problem solvers.

Because of the interaction, the problem of selecting the optimal group becomes non-trivial. In this paper, we show that the problem of selecting an optimal group is computationally difficult (NP-hard). Moreover, we will show that this problem is NP-hard already if we take into account the simplest possible non-linear terms - i.e., quadratic terms. In other words, the problem becomes NP-hard already for the following productivity expression:

$$
p=\sum_{i=1}^{n} p_{i} \cdot x_{i}+\sum_{i \neq j}^{n} p_{i j} \cdot x_{i} \cdot x_{j} .
$$

So, we arrive at the following problem.

\section{Definitions, Main Results, And ExAmple}

\section{A. Definitions}

Definition 1. By a problem of selecting the most efficient group, we mean the following problem. We are given:

- an integer $n>0$;

- rational numbers $p_{1}, \ldots, p_{n}$, and

- rational numbers $r_{i j}, 1 \leq i, j \leq n, i \neq j$.

We must find the combination of $n$ values $x_{1} \in\{0,1\}, \ldots, x_{n} \in\{0,1\}$ for which the expression

$$
p=\sum_{i=1}^{n} p_{i} \cdot x_{i}+\sum_{i \neq j} p_{i j} \cdot x_{i} \cdot x_{j}
$$

is the largest possible.

Instead of trying to find the most efficient group, we can also formulate a less ambitious problem of finding a group with a given efficiency.

Definition 2. By a problem of selecting a group with a given efficiency, we mean the following problem. We are given:

- an integer $n>0$;

- rational numbers $p_{1}, \ldots, p_{n}$,

- rational numbers $r_{i j}, 1 \leq i, j \leq n, i \neq j$, and

- a rational value $p_{0}$.

We must find the combination of $n$ values

$x_{1} \in\{0,1\}, \ldots, x_{n} \in\{0,1\}$ for which

$$
p \stackrel{\text { def }}{=} \sum_{i=1}^{n} p_{i} \cdot x_{i}+\sum_{i \neq j} p_{i j} \cdot x_{i} \cdot x_{j} \geq p_{0} .
$$

\section{B. Main results}

Proposition 1. The problem of selecting the most efficient group is NP-hard.

Proposition 2. The problem of selecting a group with a given efficiency is NP-hard.

Comment. The proof of this result is placed in the special Proofs section.

For readers who are not very familiar with the notion of NP-hardness and of Np-hardness proofs, we precede the Proof section with a special section describing the NPhardness notions and ideas. Readers who are familiar with these notions and ideas can skip this preceding section and go directly to the Proofs section. 


\section{Examples}

For simplicity, let us assume that we have two groups of people. People from each groups are equally productive, and their productivity increases when they team together with people from the same group. On the other hand, due to cultural differences, interaction between people from different groups is not as productive. Examples of such groups are easy to find: e.g., in big projects where, in principle, problems can be solved both by an appropriate hardware and by an appropriate software, hardware and software folks often experience difficulties communicating with other, and these difficulties can drag down the collective performance.

Let us assume that a company has $m$ folks from one group; (we will assign them indices $i=1, \ldots, m$ ), and the same company has the same number $m$ of folks from the second group (with indices $i=m+1, \ldots, 2 m$ ). Let us assume that the productivity $p_{i}$ of every person from both groups is the same, i.e., that $p_{1}=\ldots=p_{m}=p_{m+1}=\ldots=p_{2 m}=1$. Let us also assume that each collaboration between people from the same groups adds one extra unit to the overall productivity, e.g., $p_{i j}=p_{m+i, m+j}=1$ for all $i, j=$ $1, \ldots, m$. Let $a$ denote the loss of productivity caused by a tension between the two representatives of opposite groups, i.e., that $p_{i, m+j}=p_{m+i, j}=-a$ for all $i, j=1, \ldots, m$. Our objective is to select a group consisting of some (maybe none) representatives of the first group and some (maybe none) representatives of the second group so that the overall productivity of the resulting group is the largest.

In this simplified situation, in terms of productivity, all the persons from the first groups are equivalent - and all the persons from the second group are also equivalent to each other. Thus, the overall productivity does not depend on which exactly persons from the first group we select, and it does not depend on who exactly we select from the second group. The overall productivity only depends on the number of people $m_{1}$ chosen from the first group and on the number of people $m_{2}$ chosen from the second group.

If we only select $m_{1}$ people from the first group (i.e., if we choose $m_{2}=0$ ), then the productivity becomes equal to

$p=\sum_{i=1}^{n} p_{i} \cdot x_{i}+\sum_{i \neq j}^{n} p_{i j} \cdot x_{i} \cdot x_{j}=m_{1}+m_{1} \cdot\left(m_{1}-1\right)=m_{1}^{2}$.

Similarly, if we only select $m_{2}$ people from the second group (i.e., if we choose $m_{1}=0$ ), then the overall productivity is equal to $m_{2}^{2}$. In the general case, we have

$p=\sum_{i=1}^{n} p_{i} \cdot x_{i}+\sum_{i \neq j}^{n} p_{i j} \cdot x_{i} \cdot x_{j}=m_{1}^{2}+m_{2}^{2}-a \cdot m_{1} \cdot m_{2}$.

For example, if we select one person from each group, i.e., if we take $m_{1}=m_{2}=1$, then the overall productivity is $p=1^{2}+1^{2}-a \cdot 1 \cdot 1=2-a$. If we select two people from each group, then the overall productivity is $p=2^{2}+2^{2}-a \cdot 2 \cdot 2=$ $8-4 a$. The larger $2+2$ group is more productive than the smaller $1+1$ group if $8-4 a>2-a$, i.e., if $6>3 a$ and $a<2$. When $a=2$, these two groups are equally productive, and when $a>2$, the smaller group is more productive.
In other words, if the coefficient $a$ that describes the tension between the groups is not too high (smaller than the threshold $a=2$ ), then it is more beneficial to use a larger group (in spite of this tension). On the other hand, if the tension is too high (exceeds the threshold), then larger groups are no longer more productive.

A similar threshold $a=1$ appears when we compare the "maximally diverse" group $m_{1}=m_{2}=m$ and the "minimally diverse" groups (either $m_{1}=m$ and $m_{2}=0$ or $m_{1}=m$ and $\left.m_{2}=0\right)$. Indeed, in the first case, we have $p=m^{2}+m^{2}-a \cdot m^{2}=(2-a) \cdot m^{2}$, while in the second case, we have $p=m^{2}$. The first value is larger than the second one when $2-a>1$, i.e., when $a<1$.

It turns out that in our simple example, these groups are actually optimal; namely:

- when $a<1$, the most productive group is the one that includes all the persons from both groups: $m_{1}=m_{2}=$ $m$;

- on the other hand, when $a>1$, then the most productive group is a one that includes all the persons from one group and no one from the other group: either $m_{1}=m$ and $m_{2}=0$ or $m_{1}=m$ and $m_{2}=0$.

For $a=1$, both the "maximally diverse" group $m_{1}=m_{2}=$ $m$ and the "minimally diverse groups" (either $m_{1}=m$ and $m_{2}=0$ or $m_{1}=m$ and $m_{2}=0$ ) are equally productive.

Indeed, we will show that the corresponding maximum is attained even when we formally allow real (nor necessarily integer) values of $m_{1} \in[0, m]$ and $m_{2} \in[0, m]$. The maximum of a differentiable function $p$ over each of the variable $m_{i}$ taking values over an interval is attained either at one of the endpoints $m_{i}=0$ or $m_{i}=m$ of this interval, or when the partial derivative relative to $m_{i}$ is equal to 0 , i.e., when $\frac{\partial p}{\partial m_{i}}=0$. Here, $\frac{\partial p}{\partial m_{1}}=2 m_{1}-a \cdot m_{2}$, so, for $m_{1}$, we have three possible values where the maximum can be attained: when $m_{1}=0$, when $m_{1}=m$, and when $2 m_{1}-a \cdot m_{2}$ (i.e., when $\left.m_{1}=(a / 2) \cdot m_{2}\right)$. Similar three cases are possible for $m_{2}$, so, to find the maximum, we have to consider $3 \times 3=9$ combinations of these cases. Due to symmetry, we only need to only consider 6 cases:

- $m_{1}=m_{2}=0$; in this case, $p=0$ - smaller than for the minimally diverse group; so this cannot be the maximum;

- $m_{1}=0$ and $m_{2}=(a / 2) \cdot m_{1}$; here too, $m_{1}=m_{2}=0$ and $p=0$;

- $m_{1}=0$ and $m_{2}=m$, where $p=m^{2}$;

- $m_{1}=(a / 2) \cdot m_{2}$ and $m_{2}=(a / 2) \cdot m_{1}$; here, $m_{1}=$ $(a / 2)^{2} \cdot m_{1}$, so either $m_{1}=m_{2}=0$ or (for $a=2$ ) $m_{1}=m_{2}$, in which case the maximum is attained when $m_{1}=m_{2}=0$ or $m_{1}=m_{2}=m$;

- $m_{1}=(a / 2) \cdot m_{2}$ and $m_{2}=m$; in this case, $p=$ $(a / 2)^{2} \cdot m^{2}+m^{2}-a \cdot(a / 2) \cdot m^{2}=(1-a / 4) \cdot m^{2}<m^{2}$, so this cannot be the maximum either;

- $m_{1}=m_{2}=m$, in which case $p=(2-a) \cdot m^{2}$.

Thus, the maximum is either when $m_{1}=m_{2}=m$ or when $m_{1}=0$ and $m_{2}=m$ (or vice versa). 
Comment. In this example, we have explicitly found the optimal group. Our main result says that in general, finding the most productive group is a computationally difficult problem.

\section{WHAT IS NP-HARDNESS: A BRIEF INFORMAL REMINDER}

As we have mentioned earlier, the intent of this section is that the readers who are not very familiar with NP-hardness and related notions will be able to understand our proofs. Readers who are already well familiar with NP-hardness and related notions can skip this section and go directly to the next (Proofs) section.

Informally, a problem $\mathcal{P}_{0}$ is called $N P$-hard if it is at least as hard as all other problems from a certain reasonable class. Let us describe this notion in more detail.

\section{A. When is an Algorithm Feasible?}

The notion of NP-hardness is related to the known fact that some algorithms are feasible and some are not. Whether an algorithm is feasible or not depends on how many computational steps it needs.

For example, if for some input $x$ of length $\operatorname{len}(x)=n$, an algorithm requires $2^{n}$ computational steps, then for an input of a reasonable length $n \approx 300$, we would need $2^{300}$ computational steps. Even if we use a hypothetical computer for which each step takes the smallest physically possible time (the time during which light passes through the smallest known elementary particle), we would still need more computational steps than can be performed during the (approximately 20 billion years) lifetime of our Universe.

A similar estimate can be obtained for an arbitrary algorithm whose running time $t(n)$ on inputs of length $n$ grows at least as an exponential function, i.e., for which, for some $c>0, t(n) \geq \exp (c \cdot n)$ for all $n$. As a result, such algorithms (called exponential-time) are usually considered not feasible.

Comment. The fact that an algorithm is not feasible, does not mean that it can never be applied: it simply means that there are cases when its running time will be too large for this algorithm to be practical; for other inputs, this algorithm can be quite useful.

On the other hand, if the running time grows only as a polynomial of $n$ (i.e., if an algorithm is polynomial-time), then the algorithm is usually quite feasible.

As a result of the above two examples, researchers have arrived at the following idea: An algorithm $\mathcal{U}$ is called feasible if and only if it is polynomial-time, i.e., if and only if there exists a polynomial $P(n)$ such that for every input $x$ of length len $(x)$, the computational time $t_{\mathcal{U}}(x)$ of the algorithm $\mathcal{U}$ on the input $x$ is bounded by $P(\operatorname{len}(x))$ : $t_{\mathcal{U}}(x) \leq P(\operatorname{len}(x))$.

In most practical cases, this idea adequately describes our intuitive notion of feasibility: polynomial-time algorithms are usually feasible, and non-polynomial-time algorithms are usually not feasible. However, the reader should be warned that in some (rare) cases, it does not work:

- Some algorithms are polynomial-time but not feasible: e.g., if the running time of an algorithm is $10^{300} \cdot n$, this algorithm is polynomial-time, but, clearly, not feasible.

- Vice versa, there exist algorithms whose computation time grows, say, as $\exp (0.000 \ldots 01 \cdot \operatorname{len}(x))$. Legally speaking, such algorithms are exponential time and thus, not feasible, but for all practical purposes, they are quite feasible.

It is therefore desirable to look for a better formalization of feasibility, but as of now, "polynomial-time" is the best known description of feasibility.

Definition 3. An algorithm $U$ is called feasible if there exists a polynomial $P(n)$ such that for every input $x$, the running time $t_{U}(x)$ of this algorithm does not exceed $P(\operatorname{len}(x))$, where by $\operatorname{len}(x)$, we denoted the length of the input $x$ (i.e., the number of bits that form this input).

\section{B. When is a Problem Tractable?}

At first glance, now, that we have a definition of a feasible algorithm, we can describe which problems are tractable and which problems are intractable: If there exists a polynomialtime algorithm that solves all instances of a problem, this problem is tractable, otherwise, it is intractable.

In some cases, this ideal solution is possible, and we either have an explicit polynomial-time algorithm, or we have a proof that no polynomial-time algorithm is possible.

Unfortunately, in many cases, we do not know whether a polynomial-time algorithm exists or not. This does not mean, however, that the situation is hopeless: instead of the missing ideal information about intractability, we have another information that is almost as good.

Namely, for some cases, we do not know whether the problem can be solved in polynomial time or not, but we do know that this problem is as hard as practical problems can get: if we can solve this problem easily, then we would have an algorithm that solves all problems easily, and the existence of such universal solves-everything-fast algorithm is very doubtful. We can, therefore, call such "hard" problems intractable.

In order to formulate this notion in precise terms, we must describe what we mean by a problem, and what we mean by the ability to reduce other problems to this one.

What is a practical problem? When we say that there is a practical problem, we usually mean that:

- we have some information (we will denote its computer representation by $x$ ), and

- we know the relationship $R(x, y)$ between the known information $x$ and the desired object $y$.

In the computer, everything is represented by a binary sequence (i.e., sequence of 0's and 1's), so we will assume that $x$ and $y$ are binary sequences.

In this section, we will trace all the ideas on two examples, one taken from mathematics and one taken from physics. 
- (Example from mathematics) We are given a mathematical statement $x$. The desired object $y$ is either a proof of $x$, or a "disproof" of $x$ (i.e., a proof of "not $x$ "). Here, $R(x, y)$ means that $y$ is a proof either of $x$, or of "not $x$ ".

- (Example from physics) $x$ is the results of the experiments, and the desired $y$ is the formula that fits all these data. Imagine that we have a series of measurements of voltage and current: e.g., $x$ consists of the following pairs $\left(x_{1}^{(k)}, x_{2}^{(k)}\right), 1 \leq k \leq 10$ : $(1.0,2.0),(2.0,4.0), \ldots,(10.0,20.0)$; we want to find a formula that is consistent with these experiments (e.g., $y$ is the formula $x_{2}=2 \cdot x_{1}$ ).

For a problem to be practically meaningful, we must have a way to check whether the proposed solution is correct. In other words, we must assume that there exists a feasible algorithm that checks $R(x, y)$ (given $x$ and $y$ ). If no such feasible algorithm exists, then there is no criterion to decide whether we achieved a solution or not.

Another requirement for a real-life problem is that in such problems, we usually know an upper bound for the length len $(y)$ of the description of $y$. In the above examples:

- In the mathematical problem, a proof must be not too huge, else it is impossible to check whether it is a proof or not.

- In the physical problem, it makes no sense to have a formula $x_{2}=f\left(x_{1}, C_{1}, \ldots, C_{40}\right)$ with, say, 40 parameters to describe the results $\left(x_{1}^{(1)}, x_{2}^{(1)}\right), \ldots,\left(x_{1}^{(10)}, x_{2}^{(10)}\right)$ of 10 experiments, for two reasons:

- First, one of the goals of physics is to discover the laws of nature. If the number of parameters exceeds the number of experimental data, then no matter what dependency $f\left(x_{1}, C_{1}, \ldots\right)$ we choose, in order to determine $C_{i}$, we have, say, 10 equations with 40 unknowns. Such under-determined system usually has a solution, so the fact that, say, a linear formula with many parameters fits all the experimental data does not mean that the dependency is proven to be linear: a quadratic or cubic formula with as many parameters will fit the same data as well.

- Second, another goal of physics (definitely related to the first one) is to find a way to compress the data, so that we will not need to store all billions of experimental results in order to make predictions. A dependency $y$ that requires more storage space than the original data $x$ is clearly not satisfying this goal.

In all cases, it is necessary for a user to be able to read the desired solution symbol-after-symbol, and the time required for that reading must be feasible. In the previous section, we have formalized "feasible time" as a time that is bounded by some polynomial of len $(x)$. The reading time is proportional to the length len $(y)$ of the answer $y$. Therefore, the fact the reading time is bounded by a polynomial of len $(x)$ means that the length of the output $y$ is also bounded by some polynomial of len $(x)$, i.e., that $\operatorname{len}(y) \leq P_{L}(\operatorname{len}(x))$ for some polynomial $P_{L}$.

So, we arrive at the following formulation of a problem:

Definition 4. By a general practical problem (or simply a problem, for short), we mean a pair $\left\langle R, P_{L}\right\rangle$, where $R(x, y)$ is a feasible algorithm that transforms two binary sequences into a Boolean value ("true" or "false"), and $P_{L}$ is a polynomial.

Definition 5. By an instance of a (general) problem $\left\langle R, P_{L}\right\rangle$, we mean the following problem:

GIVEN: a binary sequence $x$. GENERATE

- either $y$ such that $R(x, y)$ is true and $\operatorname{len}(y) \leq$ $P_{L}(\operatorname{len}(x))$,

- $o r$, if such a y does not exist, a message saying that there are no solutions.

For example, for the general mathematical problem described above, an instance would be: given a statement, find its proof or disproof.

Comments. What we called "general practical problems" is usually described as "problems from the class NP" (to separate them from more complicated problems in which the solution may not be easily verifiable). Problems for which there is a feasible algorithm that solves all instances are called tractable, easily solvable, or "problems from the class P" (P from Polynomial). It is widely believed that not all (general practical) problems are easily solvable (i.e., that $\mathrm{NP} \neq \mathrm{P}$ ), but it has never been proved.

One way to solve an NP problem is to check $R(x, y)$ for all binary sequences $y$ with $\operatorname{len}(y) \leq P_{L}(\operatorname{len}(x))$. This algorithm (called British Museum algorithm) requires $2^{P_{L}(\text { len }(x))}$ checks. This algorithm takes exponential time and is therefore, not feasible.

\section{Reducing a Problem to Another One}

Let us start with an example. Suppose that we can have an algorithm that checks whether a given system of linear inequalities

$$
a_{i 1} \cdot x_{1}+\ldots+a_{i m} \cdot x_{m} \geq b_{i}, \quad 1 \leq i \leq n,
$$

with known $a_{i j}$ and $b_{i}$, has a solution. A problem of checking whether a given system of inequalities and equalities $c_{k 1}$. $x_{1}+\ldots+c_{k m} \cdot x_{m}=d_{k}$ is consistent can be reduced to the problem of checking inequalities if we replace each equality by two inequalities: $c_{k 1} \cdot x_{1}+\ldots+c_{k m} \cdot x_{m} \geq d_{k}$ and $\left(-c_{k 1}\right) \cdot x_{1}+\ldots+\left(-c_{k m}\right) \cdot x_{m} \geq-d_{j}$ (the latter being equivalent to $\left.c_{k 1} \cdot x_{1}+\ldots+c_{k m} \cdot x_{m} \leq d_{k}\right)$.

In general, we can say that a problem $\mathcal{P}=\left\langle R, P_{L}\right\rangle$ can be reduced to a problem $\mathcal{P}^{\prime}=\left\langle R^{\prime}, P_{L}^{\prime}\right\rangle$ if there exist three feasible algorithms $U_{1}, U_{2}$, and $U_{3}$ with the following properties:

- The (feasible) algorithm $U_{1}$ transforms each input $x$ of the first problem into an input of the second problem. 
- The (feasible) algorithm $U_{2}$ transforms each solution $y$ of the first problem into the solution of the corresponding case of the second problem: i.e., if $R(x, y)$ is true, then $R^{\prime}\left(U_{1}(x), U_{2}(y)\right)$ is also true.

- The (feasible) algorithm $U_{3}$ transforms each solution $y^{\prime}$ of the corresponding instance of the second problem into the solution of the first problem: i.e., if $R^{\prime}\left(U_{1}(x), y^{\prime}\right)$ is true, then $R\left(x, U_{3}\left(y^{\prime}\right)\right)$ is also true.

(In the above example, $U_{1}$ transforms each equality into two inequalities, and $U_{2}$ and $U_{3}$ simply do not change the values $x_{i}$ at all.)

If there exists a reduction, then an instance $x$ of the first problem is solvable if and only if the corresponding instance $U_{1}(x)$ of the second problem is solvable. Moreover, if we can actually solve the second instance (and find a solution $y^{\prime}$ ), we will then be able to find a solution to the original instance $x$ of the first problem (as $U_{3}\left(y^{\prime}\right)$ ). Thus, if we have a feasible algorithm for solving the second problem, we would thus design a feasible algorithm for solving the first problem as well.

Comment. We only described the simplest way of reducing one problem to another one: when a single instance of the first problem is reduced to a single instance of the second problem. In some cases, we cannot reduce to a single case, but we can reduce to several cases, solving which helps us solve the original instance of the first problem.

\section{Definition 6.}

- A problem (not necessarily from the class $N P$ ) is called NP-hard if every problem from the class NP can be reduced to it.

- If a problem from the class NP is NP-hard, it is called NP-complete.

If a problem $\mathcal{P}$ is NP-hard, then every feasible algorithm for solving this problem $\mathcal{P}$ would lead to feasible algorithms for solving all problems from the class NP, and this is generally believed to be hardly possible.

- For example, mathematicians believe that not only there is no algorithm for checking whether a given statement is provable or not (the famous Gödel's theorem has proven that), but also they believe that there is no feasible way to find a proof of a given statement even if we restrict the lengths of possible proofs. (In other words, mathematicians believe that computers cannot completely replace them.)

- Similarly, physicists believe that what they are doing cannot be completely replaced by computers.

In view of this belief, NP-hard problems are also called intractable.

Comment. It should be noted that although most scientists believe that intractable problems are not feasible, we still cannot prove (or disprove) this fact. If a NP-hard problem can be solved by a feasible algorithm, then (by definition of NP-hardness) all problems from the class NP will be solvable by feasible algorithms and thus, $\mathrm{P}=\mathrm{NP}$. Vice versa, if $\mathrm{P}=\mathrm{NP}$, then all problems from the class $\mathrm{NP}$ (including all NP-complete problems) can be solved by polynomial-time (feasible) algorithms.

So, if $\mathrm{P} \neq \mathrm{NP}$ (which is a common belief), then the fact that the problem is NP-hard means that no matter what algorithm we use, there will always be some cases for which the running time grows faster than any polynomial. Therefore, for these cases, the problem is truly intractable.

\section{Examples of NP-Hard Problems}

Historically the NP-complete problem proved to be NPcomplete was the so-called propositional satisfiability (3$S A T)$ problem for $3-\mathrm{CNF}$ formulas.

This problem consists of the following: Suppose that an integer $v$ is fixed, and a formula $F$ of the type $F_{1} \& F_{2} \& \ldots \& F_{k}$ is given, where each of the expressions $F_{j}$ has the form $a \vee b$ or $a \vee b \vee c$, and $a, b, c$ are either the variables $z_{1}, \ldots, z_{v}$, or their negations $\neg z_{1}, \ldots, \neg z_{v}$ (these $a, b, c, \ldots$ are called literals)

For example, we can take a formula $\left(z_{1} \vee \neg z_{2}\right) \&\left(\neg z_{1} \vee\right.$ $\left.z_{2} \vee \neg z_{3}\right)$.

If we assign arbitrary Boolean values ("true" or "false") to $v$ variables $z_{1}, \ldots, z_{v}$, then, applying the standard logical rules, we get the truth value of $F$. We say that a formula $F$ is satisfiable if there exist truth values $z_{1}, \ldots, z_{v}$ for which the truth value of the expression $F$ is "true". The problem is: given $F$, check whether it is satisfiable.

In the subset sum problem, given $n$ integers $s_{1}, \ldots, s_{n}$, we must check whether there exist values $x_{1}, \ldots, x_{n} \in\{-1,1\}$ for which $s_{1} \cdot x_{1}+\ldots+s_{n} \cdot x_{n}=0$.

\section{E. How NP-Hardness Is Usually Proved}

The original proof of NP-hardness of certain problems $\mathcal{P}_{0}$ is rather complex, because it is based on explicitly proving that every problem from the class NP can be reduced to the problem $\mathcal{P}_{0}$. However, once we have proven NP-hardness of a problem $\mathcal{P}_{0}$, the proof of NP-hardness of other problems $\mathcal{P}_{1}$ is much easier.

Indeed, from the above description of a reduction, one can easily see that reduction is a transitive relation: if a problem $\mathcal{P}$ can be reduced to a problem $\mathcal{P}_{0}$, and the problem $\mathcal{P}_{0}$ can be reduced to a problem $\mathcal{P}_{1}$, then, by combining these two reductions, we can prove that $\mathcal{P}$ can be reduced to $\mathcal{P}_{1}$.

Thus, to prove that a new problem $\mathcal{P}_{1}$ is NP-hard, it is sufficient to prove that one of the known NP-hard problems $\mathcal{P}_{0}$ can be reduced to this problem $\mathcal{P}_{1}$. Indeed, since $\mathcal{P}_{0}$ is NP-hard, every other problem $\mathcal{P}$ from the class NP can be reduced to this problem $\mathcal{P}_{0}$. Since $\mathcal{P}_{0}$ can be reduced to $\mathcal{P}_{1}$, we can now conclude, by transitivity, that every problem $\mathcal{P}$ from the class NP can be reduced to this problem $\mathcal{P}_{1}-$ i.e., that the problem $\mathcal{P}_{1}$ is indeed NP-hard.

Comment. As a consequence of the definition of NPhardness, we can conclude that if a problem $\mathcal{P}_{0}$ is NP-hard, then every more general problem $\mathcal{P}_{1}$ is also NP-hard.

Indeed, the fact that $\mathcal{P}_{0}$ is NP-hard means that every instance $p$ of every problem $\mathcal{P}$ can be reduced to some 
instance $p_{0}$ of the problem $\mathcal{P}_{0}$. Since the problem $\mathcal{P}_{1}$ is more general than the problem $\mathcal{P}_{0}$, every instance $p_{0}$ of the problem $\mathcal{P}_{0}$ is also an instance of the more general problem $\mathcal{P}_{1}$.

Thus, every instance $p$ of every problem $\mathcal{P}$ can be reduced to some instance $p_{0}$ of the problem $\mathcal{P}_{1}-$ i.e., that the more general problem $\mathcal{P}_{1}$ is indeed NP-hard.

\section{PROOFS}

\section{A. Reduction in Our Proof: to Subset Sum, a Known NP-}

\section{Hard Problem}

We prove NP-hardness of our problem by reducing a known NP-hard problem to it: namely, a subset sum problem, in which we are given $n$ positive integers $s_{1}, \ldots, s_{n}$, and we must find the signs $\varepsilon_{i} \in\{-1,1\}$ for which

$$
\sum_{i=1}^{n} \varepsilon_{i} \cdot s_{i}=0
$$

see, e.g., [5], [10].

A reduction means that to every instance $s_{1}, \ldots, s_{n}$ of the subset sum problem, we must assign (in a feasible, i.e., polynomial-time way) an instance of our problem in such a way that the solution to the new instance will lead to the solution of the original instance.

\section{B. Reduction: Idea}

In our reduction, we would like to transform each variable $\varepsilon_{i}$ from the subset sum problem into a variable $x_{i}$ from our problem, so that our problem (formulated in terms of $x_{i}$ ) is optimal if and only if the original problem has a solution.

For that, we need to transform each variable $x_{i}$ which takes the values 0 and 1 into a variable $\varepsilon_{i}$ that takes values -1 and 1 (and vice versa). The simplest way to perform this reduction is to take a linear function $\varepsilon_{i}=a \cdot x_{i}+b$, where the coefficients $a$ and $b$ are selected in such as way that $a \cdot 0+b=-1$ and $a \cdot 1+b=1$. In other words, we have $b=-1$ and $a+b=1$. Substituting $b=-1$ into the equation $a+b=1$, we conclude that $a=2$, i.e., that

$$
\varepsilon_{i}=2 \cdot x_{i}-1 \text {. }
$$

Let us select an integer $p_{0}>0$ and consider the formula

$$
p=p_{0}-\left(\sum_{i=1}^{n} \varepsilon_{i} \cdot s_{i}\right)^{2} .
$$

This expression is always $\leq p_{0}$, and it attains the value $p_{0}$ if and only if $\sum_{i=1}^{n} \varepsilon_{i} \cdot s_{i}=0$. In terms of $x_{i}$, we have

$$
p=p_{0}-\left(\sum_{i=1}^{n}\left(2 \cdot x_{i}-1\right) \cdot s_{i}\right)^{2}
$$

i.e.,

$$
p=p_{0}-\left(\sum_{i=1}^{n} x_{i} \cdot\left(2 \cdot s_{i}\right)-s_{0}\right)^{2}
$$

where we denoted

$$
s_{0} \stackrel{\text { def }}{=} \sum_{i=1}^{n} s_{i} .
$$

By using the formula for the square of the difference, we conclude that

$$
\begin{gathered}
p=p_{0}-\left(\sum_{i=1}^{n} x_{i} \cdot\left(2 \cdot s_{i}\right)\right)^{2}+ \\
2 \cdot s_{0} \cdot \sum_{i=1}^{n} x_{i} \cdot\left(2 \cdot s_{i}\right)-s_{0}^{2},
\end{gathered}
$$

i.e.,

$$
\begin{gathered}
p=p_{0}-\left(\sum_{i=1}^{n} x_{i} \cdot\left(2 \cdot s_{i}\right)\right)^{2}+ \\
\sum_{i=1}^{n} x_{i} \cdot\left(4 \cdot s_{0} \cdot s_{i}\right)-s_{0}^{2} .
\end{gathered}
$$

The square of the sum takes the form

$$
\begin{gathered}
\left(\sum_{i=1}^{n} x_{i} \cdot\left(2 \cdot s_{i}\right)\right)^{2}= \\
\sum_{i=1}^{n} x_{i}^{2} \cdot\left(4 \cdot s_{i}^{2}\right)+\sum_{i \neq j}\left(4 \cdot s_{i} \cdot s_{j}\right) \cdot x_{i} \cdot x_{j} .
\end{gathered}
$$

Since $x_{i}=0$ or $x_{i}=1$, we always have $x_{i}^{2}=x_{i}$ and thus,

$$
\begin{gathered}
\left(\sum_{i=1}^{n} x_{i} \cdot\left(2 \cdot s_{i}\right)\right)^{2}= \\
\sum_{i=1}^{n} x_{i} \cdot\left(4 \cdot s_{i}^{2}\right)+\sum_{i \neq j}\left(4 \cdot s_{i} \cdot s_{j}\right) \cdot x_{i} \cdot x_{j} .
\end{gathered}
$$

Substituting this expression into the above formula for $p$, we get

$$
\begin{gathered}
p=p_{0}-\sum_{i=1}^{n} x_{i} \cdot\left(4 \cdot s_{i}^{2}\right)-\sum_{i \neq j}\left(4 \cdot s_{i} \cdot s_{j}\right) \cdot x_{i} \cdot x_{j}+ \\
\sum_{i=1}^{n} x_{i} \cdot\left(4 \cdot s_{0} \cdot s_{i}\right)-s_{0}^{2} .
\end{gathered}
$$

By grouping together terms independent on $x_{i}$ and terms proportional to $p_{i}$, we get

$$
\begin{gathered}
p=\left(p_{0}-s_{0}^{2}\right)+\sum_{i=1}^{n} x_{i} \cdot\left(4 \cdot s_{0} \cdot s_{i}-4 \cdot s_{i}^{2}\right)+ \\
\sum_{i \neq j}\left(-4 \cdot s_{i} \cdot s_{j}\right) \cdot x_{i} \cdot x_{j} .
\end{gathered}
$$

Thus, if we choose

$$
p_{0}=s_{0}^{2},
$$

then the above expression takes the desired form

$$
p=\sum_{i=1}^{n} p_{i} \cdot x_{i}+\sum_{i \neq j} p_{i j} \cdot x_{i} \cdot x_{j},
$$


with

$$
p_{i}=4 \cdot s_{0} \cdot s_{i}-4 \cdot s_{i}^{2}
$$

and

$$
p_{i j}=-4 \cdot s_{i} \cdot s_{j}
$$

\section{Resulting Reduction}

To each particular case of the subset sum problem, described by parameters $s_{1}, \ldots, s_{n}$, we assign the following particular case of our problem. First, we compute

$$
p_{0}=s_{0}=\sum_{i=1}^{n} s_{i}
$$

then, we compute

$$
p_{i}=4 \cdot s_{0} \cdot s_{i}-4 \cdot s_{i}^{2} ; \quad p_{i j}=-4 \cdot s_{i} \cdot s_{j} .
$$

For these values, the quadratic function

$$
p=\sum_{i=1}^{n} p_{i} \cdot x_{i}+\sum_{i \neq j} p_{i j} \cdot x_{i} \cdot x_{j}
$$

has the form

$$
p=p_{0}-\left(\sum_{i=1}^{n} \varepsilon_{i} \cdot s_{i}\right)^{2},
$$

where $\varepsilon_{i}=2 \cdot x_{i}-1 \in\{-1,1\}$.

The above argument shows that for this selection, the quadratic function $p$ attains the value $p_{0}=s_{0}$ if and only if the original instance of the subset sum problem $\sum_{i=1}^{n} \varepsilon_{i} \cdot s_{i}=0$ has a solution with $\varepsilon_{i} \in\{-1,1\}$, and thus, with

$$
x_{i}=\frac{\varepsilon_{i}+1}{2} \in\{0,1\} .
$$

The reduction is proven, so our problem is indeed NP-hard.

Comment. Strictly speaking, we have proved NP-hardness of a specific choice of the quadratic function $p\left(x_{1}, \ldots, x_{n}\right)$. However, we have already mentioned earlier that if a problem $\mathcal{P}_{0}$ is NP-hard, then a more general problem $\mathcal{P}_{1}$ is NP-hard as well. Thus, we have indeed proved that the (more general) problem is also NP-hard.

\section{CONClusions}

One of the applications of fuzzy techniques is to formalize the meaning of words from natural language such as "efficient", "diverse", etc. The main idea behind fuzzy techniques is that they formalize expert knowledge expressed by words from natural language; see, e.g, [4], [8].

In this paper, we have shown that if we do not use this knowledge, i.e., if we only use the data, then selecting the most efficient group (or even selecting a group with a given efficiency) becomes a computationally difficult (NP-hard) problem. Thus, the need to select such groups in reasonable time justifies the use of fuzzy (intelligent) techniques - and, moreover, the need to combine intelligent techniques with more traditional optimization techniques.

\section{REFERENCES}

[1] J.E. Gamez, F. Modave, and O. Kosheleva, "Selecting the Most Representative Sample is NP-Hard: Need for Expert (Fuzzy) Knowledge", Proceedings of the IEEE World Congress on Computational Intelligence WCCI'2008, Hong Kong, China, June 1-6, 2008, pp. 1069 1074.

[2] L. Hong and S.E. Page, "Problem solving by heterogeneous agents", Journal of Economic Theory, vol. 97, No. 1, pp. 123-163, 2001.

[3] L. Hong and S.E. Page, "Groups of diverse problem solvers can outperform groups of high-ability problem solvers", Proceedings of the National Academy of Sciences, vol. 101, no. 46. pp. 16385-16389, 2004.

[4] G. Klir and B. Yuan, Fuzzy sets and fuzzy logic: theory and applications. Prentice Hall, Upper Saddle River, New Jersey, 1995.

[5] V. Kreinovich, A. Lakeyev, J. Rohn, and P. Kahl, Computational complexity and feasibility of data processing and interval computations, Kluwer, Dordrecht, 1998.

[6] C.C. Kuo, F. Glover, and K.S. Dhir, "Analyzing and modeling the maximum diversity problem by zero-one programming", Decision Sciences, vol. 24, no. 6, pp. 1171-1185, 1993.

[7] J.H. Miller and S.E. Page, Complex Adaptive Social Systems: The Interest in Bteween, Princeton University Press, Princeton, New Jersey, 2006.

[8] H.T. Nguyen and E.A. Walker, A first course in fuzzy logic, CRC Press, Boca Raton, Florida, 2005.

[9] S.E. Page, The Difference: How the Power of Diversity Creates Better Groups, Firms, Schools, and Societies, Princeton University Press, Princeton, New Jersey, 2007.

[10] C.H. Papadimitriou, Computational Complexity, Addison Wesley, San Diego, 1994. 